\title{
Protective effect of pravastatin on doxorubicin-induced hepatotoxicity
}

\author{
Mansouri $\mathrm{E}^{1}$, Jangaran $\mathrm{A}^{2}$, Ashtari $\mathrm{A}^{3}$ \\ Cellular and Molecular Research Center, Department of Anatomical Sciences, Faculty of Medicine, \\ Ahvaz Jundishapur University of Medical Sciences, Ahvaz, Iran.dr_atefeh_ashtari@yahoo.com
}

\section{ABSTRACT}

OBJECTIVE: The present study was designed to investigate the possible protection of pravastatin against hepatic oxidative stress and dysfunctions induced by doxorubicin in rats.

BACKGROUND: Statins have beneficial effects on oxidative stress and inflammation.

METHODS: Male Sprague-Dawley rats were divided into four groups. Control group (received saline orally), Group 2 received pravastatin $(20 \mathrm{mg} / \mathrm{kg}$, i.p. for 15 days), Group 3 received single dose doxorubicin (15 mg/kg, i.p.), Group 4 was treated with pravastatin ( $20 \mathrm{mg} / \mathrm{kg}$, i.p.) daily from 5 days before to 10 days after injection of doxorubicin (15 mg/kg, i.p.). Hepatic toxicity was estimated by biochemical parameters and oxidative stress and histopathological studies.

RESULTS: Administration of doxorubicin indicated an increase in ALT, AST, ALP, TG, cholesterol, LDL and total bilirubin levels $(p<0.01)$. Doxorubicin caused a reduction in HDL and albumin levels $(p<0.01)$ as well as superoxide dismutase, glutathione peroxidase and catalase activities $(p<0.05)$ with a concomitant increase in liver malondialdehyde $(p<0.05)$ and liver damage $(p<0.001)$. Pravastatin reduced the scale liver injury $(p<$ 0.001 ) and protected liver functions and other biochemical parameters $(p<0.01)$. Increase in malondialdehyde level associated with a reduction in antioxidant activities in the doxorubicin group was attenuated by pravastatin treatment $(p<0.05)$.

CONCLUSION: Results indicated that pravastatin has a protective effect on the liver against doxorubicin-induced hepatotoxicity in rats (Tab. 3, Fig. 2, Ref. 34). Text in PDF www.elis.sk. KEY WORDS: doxorubicin, hepatotoxicity, pravastatin, oxidative stress.

\section{Introduction}

The key public health concern around the world comes to cancer. There were about 8.2 million deaths in 2012 (1). The most common treatment applied for cancer are radiation and chemotherapy. Despite antitumoral effects of these two therapeutic modalities in controlling the primary tumor and metastasis, toxicity can be produced in normal tissues by both of them and their associated adverse effects frequently outweigh clinical advantages and worsen patients' life quality (2). During the past several decades, doxorubicin (DOX), which is also known as hydroxy daunorubicin, has been extensively applied for treating different cancer types such as hepatocellular carcinoma owing to its ability to kill

${ }^{1}$ Cellular and Molecular Research Center, Department of Anatomical Sciences, Faculty of Medicine, Ahvaz Jundishapur University of Medical Sciences, Ahvaz, Iran, ${ }^{2}$ Department of Clinical Science, Faculty of Veterinary Medicine, Shahid Chamran University, Ahvaz, Iran, and '3 Student Research Committee, Ahvaz Jundishapur University of Medical Sciences, Ahvaz, Iran

Address for correspondence: A. Ashtari, School of Medical Science, Ahvaz Jundishapur University of Medical Sciences, Golestan BLV, Ahvaz, Iran.

Phone: +98.9163028208 , Fax: +98.6133332036

Acknowledgments: This work was supported by research fund (No: 94s79) from the Deputy of Research and Technology Development of AJUMS. transformed liver cells (3). Liver injury is a moderately common adverse effect associated with DOX, which is observed in treating other cancer types by using this drug $(4,5)$. Doxorubicin-induced hepatotoxicity has been reported in several experimental studies $(6,7)$. The DOX toxicity mechanism comprised the oxidative stress status characterized by producing excessive amount of reactive oxygen species (ROS) and/or decrease in antioxidant defenses leading to a disbalance in the oxygen normal metabolism. First, a semiquinone form is generated as a result of adding an electron to the quinone moiety of DOX and then, the quinone form is quickly regenerated through reduction of molecular oxygen to ROS (8). ROS produces hydrogen peroxide $\left(\mathrm{H}_{2} \mathrm{O}_{2}\right)$ and hydroxyl radicals $\left(\mathrm{OH}^{\circ}\right)$ and these products can attack DNA, oxidize it and finally induce apoptosis in both normal and tumoral tissue cells (9). The other possible mechanism for DOX toxicity is via interference with non-enzymatic metabolic reactions that iron is involved in, and so the iron metabolism can be changed through this pathway. Consequently, DOX-iron complex can be formed through the reaction of free iron with DOX which leads to the formation of ROS (10). Statins are extensively applied clinically to drop hypercholesterolemia due to their inhibitory impact on 3-hydroxy3-methylglutaryl coenzyme A (HMG-CoA) reductase, which is a catalyst for the rate-limiting step in synthesizing the cholesterol in the liver and other tissues. Furthermore, the use of statins causes advantageous alterations in other lipid fractions as well as sig- 


\section{3-277}

nificant impacts on several measures of inflammation, oxidative stress, immunity, vascular, thrombosis and renal function (11, 12). In addition, small randomized controlled clinical trials have indicated advantageous impacts in non-cardiovascular inflammatory diseases, including multiple sclerosis and rheumatoid arthritis (13). No specific and effective therapeutic agent has been known for DOX-associated hepatotoxicity. Therefore, an urgent study is required to discover compounds with the ability to develop the chemotherapy therapeutic index and to decrease the side effects of DOX on healthy tissues with no effect on their anti-neoplasic impacts $(2,14,15)$. Hence, In the present study, we evaluated the content of tissue injury in DOX-induced hepatotoxicity and we evaluated the protective effect of pravastatin, especially, on the level of oxidative stress.

\section{Materials and methods}

Animals

Male Sprague-Dawley rats (190-200 g; $\mathrm{n}=6$ per group) were obtained from Animal House Center of Ahvaz Jundishapur University of Medical Sciences, Iran. The animals were housed in plastic cages and had free access to diet and tap water. The protocol of this study was approved by Ethics Committee of Ahvaz University of Medical Sciences. Rats were exposed to a 12-h light/dark cycle, at a room temperature of $22-25^{\circ} \mathrm{C}$.

\section{Experimental design}

The rats were divided into four groups:

Group 1: Control rats given saline orally.

Group 2: Rats administered with pravastatin orally with dose of $20 \mathrm{mg} / \mathrm{kg}$ for 15 days (16).

Group 3: Rats given a single intraperitoneal injection of doxorubicin $(15 \mathrm{mg} / \mathrm{kg})(17)$.

Group 4: Pravastatin $(20 \mathrm{mg} / \mathrm{kg}$, orally) given daily from 5 days prior to 10 days after intraperitoneal injection of doxorubicin.

At the end of the experiment, the rats were weighed and then anaesthetized by ether. Blood samples were collected from the left ventricle and the sera were prepared following centrifuging at $2500 \mathrm{rpm}$ for 20 minutes and kept frozen at $-30^{\circ} \mathrm{C}$ for various biochemical analyses. Also, the liver of each animal was rapidly removed after dissection. One part was fixed in $10 \%$ formalin for $72 \mathrm{hrs}$, and then transferred into $70 \%$ alcohol for histopathological examination. An amount of $0.5 \mathrm{~g}$ was homogenized in $5 \%(\mathrm{w} / \mathrm{v})$

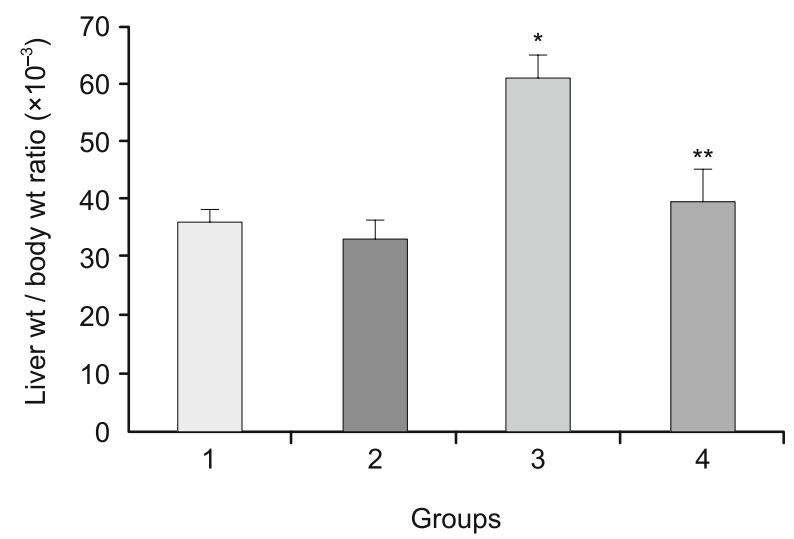

Fig. 1. Effect of pravastatin on ratio of liver weight to body weight in doxorubicin-induced hepatotoxicity in rat. * $p<0.05$ compared to groups 1and $2, * * p<0.05$ compared to group 3 .

potassium phosphate buffer (0.1 M, pH 7.4) using a homogenizer (Heidolph Silentcrosher M, Germany). The liver homogenates were centrifugated at $16,000 \times \mathrm{g}$ for $20 \mathrm{~min}$. The supernatants were used to estimation of biomarkers of oxidative stress.

\section{Biochemical evaluation}

Serum biomarkers of the liver function, including alanine transaminase (ALT), aspartate transaminase (AST), alkaline phosphatase (ALP), TG (triglyceride), Chol (cholesterol), LDL (Lowdensity lipoprotein), HDL (high-density lipoprotein), albumin, and total bilirubin (TB) were measured using commercially available kits (Pars Azmoon, Iran). All biochemical assays were performed spectrophotometrically using an autoanalyzer (Vita lab Selectra E, Netherland).

\section{Histopathological examinations}

The liver samples were embedded in paraffin and sectioned at $5 \mu \mathrm{m}$. After that, sections were stained with hematoxylin-eosin. Then, sections were examined under a light microscope. At least five microscopic fields were evaluated to score the samples. The criteria for liver damage were dilatation of sinusoids, hepatocytes vacuolization and pyknotic hepatocyte nuclei. Each sample was scored by a scale ranging from 0 to 3 ( 0 : none, 1: mild, 2: moderate, and 3: severe) for each criterion. The total score was 9 . His-

Tab. 1. Effect of pravastatin on biochemical parameters in doxorubicin-induced hepatotoxicity in rat.

\begin{tabular}{|c|c|c|c|c|}
\hline Parameters & Group 1 & Group 2 & Group 3 & Group 4 \\
\hline$\overline{\operatorname{AST}(U / 1)}$ & $170.16 \pm 5.67$ & $173.50 \pm 4.32$ & $256.33 \pm 13.66^{*}$ & $189.5 \pm 7.28 * *$ \\
\hline $\operatorname{ALT}(\mathrm{U} / \mathrm{l})$ & $76.33 \pm 5.08$ & $74.5 \pm 5.46$ & $137.5 \pm 6.77 *$ & $89.83 \pm 7.75 * *$ \\
\hline $\operatorname{ALP}(\mathrm{U} / \mathrm{l})$ & $104.83 \pm 5.11$ & $102.66 \pm 10.98$ & $188.83 \pm 7.46^{*}$ & $124.33 \pm 9.97 * *$ \\
\hline $\mathrm{TB}(\mathrm{mg} / \mathrm{dl})$ & $0.47 \pm .09$ & $0.49 \pm .09$ & $1.36 \pm 0.25 *$ & $0.65 \pm 0.13 * *$ \\
\hline Chol (g/dl) & $66.16 \pm 3.97$ & $63.33 \pm 6.37$ & $160.66 \pm 13.20 *$ & $77.33 \pm 10.68 * *$ \\
\hline TG $(\mathrm{g} / \mathrm{dl})$ & $56.5 \pm 10.09$ & $53.50 \pm 9.26$ & $89.83 \pm 6.99 *$ & $65.00 \pm 4.85 * *$ \\
\hline $\operatorname{LDL}(\mathrm{g} / \mathrm{dl})$ & $16.66 \pm 3.14$ & $15.33 \pm 2.58$ & $8.09 \pm 3.30 *$ & $4.22 \pm 1.72 * *$ \\
\hline $\mathrm{Alb}(\mathrm{g} / \mathrm{dl})$ & $4.16 \pm 0.5$ & $3.92 \pm 0.49$ & $2.85 \pm 0.4 *$ & $3.92 \pm 0.4 * *$ \\
\hline
\end{tabular}

$* \mathrm{p}<0.01$ compared to groups 1 and $2,{ }^{*} \mathrm{p}<0.01$ compared to group 3 
topathological examination and scoring were carried out by an observer unaware of the experimental groups (18).

\section{Estimation of tissue lipid peroxidation}

Tissue level of lipid peroxidase were determined by thiobarbituric acid reactive substances (TBARS) calculated as malondialdhyde (MDA) as described previously (19). The absorbance was determined at $535 \mathrm{~nm}$ against a blank using spectrophotometer and the results were expressed as (nmol/g tissue).

\section{Estimation of the antioxidant enzymes activities}

Activity of endogenous antioxidant enzymes such as superoxide dismutase (SOD), catalase (CAT) and glutathione peroxidase (GPx) were assayed using the methods described in our previous study (20). The results were expressed as U/g tissue.

\section{Statistical analysis}

All of the data are expressed as mean \pm SD. Statistical significance between groups was tested using one-way ANOVA followed by the Tukey's test. The differences were considered statistically significant when $\mathrm{p}<0.05$.

\section{Results}

Effect of pravastatin on ratio of liver weight to body weight

The ratio of liver weight to body weight in the group receiving DOX (group 3) significantly increased compared to groups 1 and $2(p<0.001)$. The ratio of liver weight to body weight in group 4 was significantly decreased compared with group 3 ( $p<0.001)$. We did not observe any significant differences between groups 1 and 2 ( $\mathrm{p}>0.05)$ (Fig. 1).

\section{Effect of pravastatin on biochemical parameters}

DOX caused a significant increase in serum ALT, AST, ALP, TG, Chol, LDL and TB levels as well as caused significantly decrease in HDL and serum albumin levels when compared to groups 1 and $2(\mathrm{p}<0.01)$. The treatment with pravastatin in group 4 markedly reversed DOX-induced increases in serum ALT, AST, TG, Chol, LDL and TB levels and the reduction in HDL and albumin levels $(p<0.01)$. These parameters in groups 1 and 2 were not significantly different from each other $(p>0.05)$ (Tab. 1).

The effect of pravastatin on histological changes and microscopic score in liver

Light microscopic evaluation of liver tissues showed that there were no pathological changes in the liver tissue in groups 1 and 2 of animals (Fig. 2 A and B). In contrast, animals receiving DOX
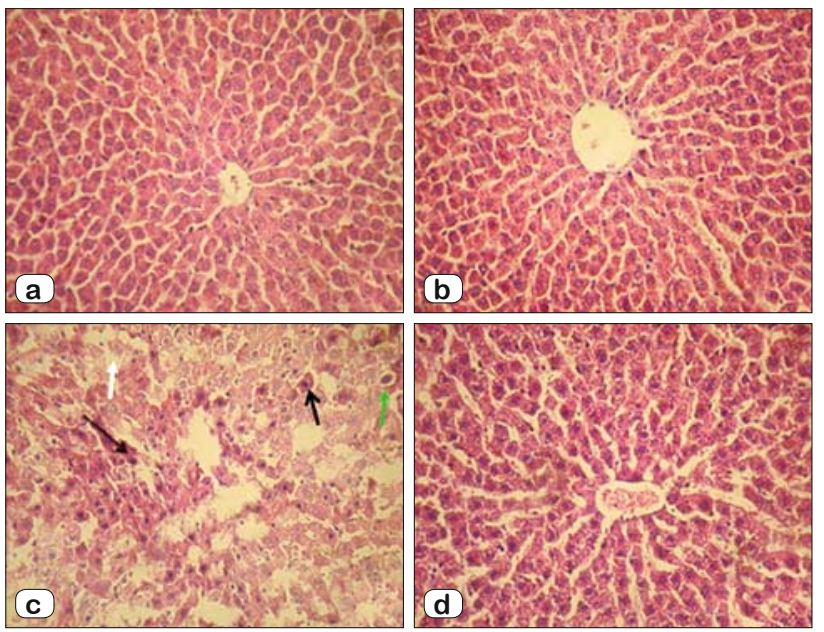

Fig. 2. Effect of pravastatin on histopathological changes in doxorubicin-induced hepatotoxicity in rat. (A) Pathological changes in the liver tissue in groups 1 (B) Pathological changes in the liver tissue in groups 2 (C) Pathological changes in the liver tissue in groups 3 (D) Pathological changes in the liver tissue in groups 4 .

revealed mild to moderate pathological changes such as degeneration of hepatocyte cords, dilatation of sinusoids, vacuolation of hepatocytes and condensation of nuclei (Fig. 2 C). Pravastatin treatment partially improved the hepatocyte cords degeneration, sinusoids, hepatocyte vacuolation which were morphologically near to control (Fig. 2 D). As a result, the microscopic score of liver tissues of the DOX-injected group were significantly higher than those of the groups 1 and $2(\mathrm{p}<0.001)$. Pravastatin treatment diminished the microscopic score effectively $(\mathrm{p}<0.001)$. There was no significant difference between groups 1 and $2(\mathrm{p}>$ 0.05) (Tab. 2).

\section{Effect of pravastatin on lipid peroxidation}

MDA level was increased significantly in the DOX -treated rats (Group 3) compared to groups 1 and $2(\mathrm{p}<0.001)$. MDA level was decreased in the pravastatin + DOX-treated group (Group 4) compared to DOX-treated group ( $<<0.001)$. However, MDA level in group 2 showed no difference compared to control group $(\mathrm{p}>0.05)$ (Tab. 3).

\section{Effect of pravastatin on antioxidant enzymes activities}

Rats treated with DOX, showed a significant reduction in activity of SOD, CAT and GPx compared to groups 1 and 2 (p $<0.05$ ). The treatment with pravastatin (Group 4) showed a significant increase in SOD $(\mathrm{p}<0.05)$, CAT $(\mathrm{p}<0.001)$, GPx $(\mathrm{p}<$

Tab. 2. Effect of pravastatin on histopathological changes in doxorubicin-induced hepatotoxicity in rat.

\begin{tabular}{lccc}
\hline Parameters & Group 1 & Group 2 & Group 3 \\
\hline Vacuolization & $0.16 \pm 0.06$ & $0.16 \pm 0.06$ & $2.66 \pm 0.21^{*}$ \\
Sinusoidal dilatation & $0.00 \pm 0.0$ & $0.00 \pm 0.0$ & $2.83 \pm 0.4^{*}$ \\
Pycnotic nuclei & $0.00 \pm 0.0$ & $0.00 \pm 0.0$ & $0.66 \pm 0.21^{* *}$ \\
\hline
\end{tabular}

$* \mathrm{p}<0.001$ compared to groups 1 and $2,{ }^{* *} \mathrm{p}<0.001$ compared to group3. 
Tab. 3. Effect of pravastatin on MDA level and SOD, GPx, CAT enzyme activities in doxorubicin induced hepatotoxicity in rat.

\begin{tabular}{lccc}
\hline Parameters & Group 1 & Group 2 & Group 3 \\
\hline MDA (nmol/g tissue) & $3.26 \pm .9$ & $3.07 \pm 1.2$ & $19.55 \pm 1.78^{*}$ \\
CAT (U/g tissue) & $289.61 \pm 31.93$ & $299.7 \pm 14.84$ & $123.78 \pm 11.48 *$ \\
GPx (U/g tissue) & $161.98 \pm 30.96$ & $184.73 \pm 39.38$ & $50.69 \pm 14.57 *$ \\
SOD (U/g tissue) & $7.18 \pm 2.41$ & $7.92 \pm 1.99$ & $1.70 \pm .81 *$ \\
\hline
\end{tabular}

$* \mathrm{p}<0.05$ compared to groups 1 and $2, * * \mathrm{p}<0.05$ compared to group 3 .

0.05 ) activities as compared to Group 3. No difference was noticeable in antioxidant enzymes activity between groups 1 and 2 $(\mathrm{p}>0.05)($ Tab 3).

\section{Discussion}

The results of the current study indicate that daily pravastatin treatment of rats dramatically ameliorates DOX-induced hepatotoxicity as affirmed by biochemical assays and microscopic evaluation. In present study, an increase in ratio of liver weight to body weight was observed in group 3 . It might be because of body weight loss caused by the loss of skeletal muscles and adipose tissue that could have occurred due to appetite loss and alterations in the metabolic rates $(21,22)$. However, a decrease in this ratio was observed for rats treated with pravastatin at the end of the period. These results are in accordance with earlier reports $(23,24)$. The current results indicate that a significant enhance in activities of ALT, AST, ALP and total bilirubin levels and a significant reduction in serum albumin resulted from administration of DOX. The drastic conditions may have increased activities of ALT, AST and ALP and these conditions resulted from doxorubicin accumulations with toxic activity in the liver and in turn this might have provoked cellular destruction or rise in the hepatic cells permeability. The enhance in the level of total bilirubin may be ascribed to mechanisms of defense versus free radical-induced oxidative injury including decreasing free radicals through enhancing electron donors such as bilirubin (4). The alterations, which are provided by DOX, are reversed by pravastatin treatment that shows the liver protective effect. The findings of recent researches are the same as our results $(11,25)$. Our results showed a significant elevation in the serum concentration of TG, Chol and LDL in the group treated with DOX, but a reduction was observed for HDL concentration in this group. These variations in the profile of lipid show that there is a possible interference of DOX with metabolism or biosynthesis of lipids (26). The elevated serum concentrations of TG, Chol and LDL as well as the reduced concentrations of HDL were successfully restored by the pravastatin treatment. At least, there is one common feature for all statins despite their pharmacokinetic profile differences: they prevent HMG-CoA converting to mevalonic acid by consecutive attenuation of the biosynthesis of cholesterol, which is related with a decrease in serum total and low-density lipoprotein (LDL) cholesterol. These features of statins are the reason of becoming the most extensively recommended lipid-lowering drugs in patients with raised serum cholesterol concentrations (27). In the current research, severe liver damage is caused by injection of DOX, as evaluated microscopically. The morphology of hepatic tissue was recognized by vacuolation of hepatocytes, degenerated hepatocytes and moderate enlargement of sinusoids. In present study, it was also indicated that there is a significant enhance in the MDA level and a reduction in the SOD, GPx and CAT activities as a result of the administration of DOX. The liver damage may take place because of the oxidative stress induced by the reactive intermediates of doxorubicin, namely semiquinone which generates from doxorubicin. The formation of radical intermediates of semiquinone by anthracyclines are reported. These radical intermediates react with molecular oxygen to form ROS which interact with cellular macromolecules and bring about cytological injury (28). In an earlier research, it was proposed that doxorubicin raised MDA level (28). In our findings, a reduction in activities of SOD, GPx and CAT in the adriamycin-induced group may have taken place because of improved generation of ROS such as superoxide and hydrogen peroxide which in turn bring about antioxidant enzymes inhibition (29). The treatment with pravastatin in part preserved the structural alterations resulting in morphology near to that of control group. Furthermore, we indicated that an elevation in the MDA level and reduction in the antioxidant enzymes activity after DOX injection was notably prevented by pravastatin. Statins are the most effective agents for dropping the cholesterol in plasma. They have also pleitropic non-lipid dependent properties, including antioxidant and anti-inflammatory (30). Moreover, the imbalance of oxidant-antioxidant is reversed by statins, which has a worthy activity of hydroxyl radical scavenging (31). These results are in accordance with results of other studies (32-34). In conclusion, pravastatin is helpful in doxorubicin-induced liver injury in rats and the mechanism of this effect may include preventing the peroxidation of lipid and preserving the antioxidant enzymes.

\section{References}

1. Ferlay J, Soerjomataram I, Dikshit $\mathbf{R}$ et al. Cancer incidence and mortality worldwide: sources, methods and major patterns in globocan 2012. Int J Cancer 2015; 136: 359-386.

2. Lamas MDJ, Nicoud MB, Sterle HA et al. Selective cytoprotective effect of histamine on doxorubicininduced hepatic and cardiac toxicity in animal models. Cell Death Discov 2015; 1: 150-159.

3. Singal JK, Iliskovic N. Doxorubicin-induced cardiomyopathy. N Engl J Med 2008; 339: 900 -905.

4. Hozayen WG, Abou Seif HS, Amin S. Protective effects of ruitn and/ or hesperidin against doxorubicin-induced hepatotoxicity. Int J Clin Nutr 2014; 2 (1): 11-17.

5. Cainelli F, Vallone A. Safety and efficacy of pegylated liposomal doxorubicin in HIV-associated Kaposi's sarcoma. Biologics 2009; 3: 385-389. 
6. Sliai AM, GH Abdel-Rahman. Protection by wheat germ oil against doxorubicininduced pathological changes and apoptosis in the kidney of male mice. Res Opin Anim Vet Sci 2014; 4 (12): 695-700.

7. Ibrahim SZ, Barakat MA, Helmy HM. Role of selenium in attenuating cardiac and hepatic damages induced by the antitumor agent, doxorubicin. Life Sci J 2010; 7 (4): 162-172.

8. Liu J, Qu W, Kadiiska MB. Role of oxidative stress in cadmium toxicity and carcinogenesis. Toxicol Appl Pharmacol 2009; 238 (3): 209-214.

9. Said M. Al-Dalaen, Aiman I. Al-Qtaitat. Review Article: Oxidative stress versus antioxidants. Am J Biosci Bioeng 2014; 2 (5): 60-71.

10. Al-Zubaidy AA, Khattab YI. The possible protective effect of pentoxifylline against doxorubicin-induced hepatotoxicity in rabbits. Int J Adv Res (2014); 2 (9): 95-101.

11. Maheshwari RA, Sailor GU, Patel L, Balaraman R. Amelioration of cisplatin-induced nephrotoxicity by statins. Indian J Pharmacol 2013; 45 (4): 354-358.

12. Pichandi S, Pasupathi P, Raoc YY et al. The role of statin drugs in combating cardiovascular diseases. Int J Cur Sci Res 2011; 1 (2): 47-56.

13. Maheshwari RA, Sailor GU, Sen AK, Balaraman R. Amelioration of cisplatin-induced hepatotoxicity by statins in rats. J Integr Health Sci 2015; 3 (1): $1-7$.

14. Octavia Y, Tocchetti CG, Gabrielson KL, Janssens S, Crijns HJ, Moens AL. Doxorubicin-induced cardiomyopathy: from molecular mechanisms to therapeutic strategies. J Mol Cell Cardiol 2012; 52: 1213-1225.

15. Citrin DE, Mitchell JB. Altering the response to radiation: sensitizers and protectors. Semin Oncol 2014; 41: 848-859.

16. Li C, Yang CW, Park JH et al. Pravastatin treatment attenuates interstitial inflammation and fibrosis in a rat model of chronic cyclosporine induced nephropathy. Am J Physiol Renal Physiol 2004; 286: 46-57.

17. El-Sheikh AAK, Morsy MA, Mahmoud MM, Rifaai RA, Abdelrahman AM. Effect of coenzyme-Q10 on doxorubicin-induced nephrotoxicity in rats. Adv Pharmacol Sci 2012; 2012: 8.

18. Ibrahim MY, Abdul AB, Abdel Wahab SI, Elhassan MM, Alzubairiand AS, Syam MM. Attenuation of cisplatin-induced hepatotoxicity in rats using zerumbone. Res J Biol Sci 2009; 4 (7): 777-784.

19. Mansouri E, Khorsandi L, Zare Moaiedi M. Grape seed proanthocyanidin extract improved some of biochemical parameters and antioxidant disturbances of red blood cells in diabetic rats. Iran J Pharm Res (2015), 14 (1): 329-334.

20. Mansouri E, Panahi M, Ghaffari MA, Ghorbani A. Effects of grape seed proanthocyanidin extract on oxidative stress induced by diabetes in rat kidney. Iran Biomed J 2011; 15 (3): 100-106.
21. Chaudhary D, Khatiwada S, Sah SK, Tamang MK, Bhattacharya S, Jha CB. Effect of doxorubicin on histomorphology of liver of wistar albino rats. J Pharm Pharmacol 2016; 4: 186-190.

22. El-Sayyad H, Ismail MF, Shalaby FM et al. Histopathological effects of cisplatin, doxorubicin and 5-flurouracil (5-FU) on the liver of male albino rats. Int J Biol Sci 2009; 5 (5): 466-473.

23. Knight SF, Yuan J, Roy S, Imig JD. Simvastatin and tempol protect against endothelial dysfunction and renal injury in a model of obesity and hypertension. Am J Physiol Renal Physiol 2010; 298: 86-94.

24. Rajitha P, Varkey J. Statins induced nephrotoxicity: a dose dependent study in albino rats. Int J Pharm Pharm Sci 2014; 6 (11): 401-406.

25. Iseri S, Ercan F, Gedik N, Yüksel M, Alican I. Simvastatin attenuates cisplatin-induced kidney and liver damage in rats. Toxicology 2007; 230: $256-264$

26. Koti BC, Vishwanathswamy AH, Wagawade J, Thippeswamy AH. Cardioprotective effect of lipistat against doxorubicin induced myocardial toxicity in albino rats. Indian J Exp Biol 2009; 47 (1): 41-46.

27. Bonetti PO, Lerman LO, Napoli $C$ et al. Statin effects beyond lipid lowering — are they clinically relevant? Eur Heart J 2003; 24: 225-248.

28. Shivakumar P, Rani MU, Reddy AG, Anjaneyulu Y. A study on the toxic effects of doxorubicin on the histology of certain organs. Toxicol Int 2012; 19 (3): 241-244.

29. Bilginoğlu A, Aydin D, Ozsoy S, Aygün H. Protective effect of melatonin on adriamycin-induced cardiotoxicity in rats. Turk Kardiyol Dern Ars 2014; 42 (3): 265-273.

30. Khoshnoud MJ, Moghbel BNA, Geramizadeh B, Niknahad H. Effect of simvastatin on cisplatin-induced nephrotoxicity in male rats. Iran J Pharm Sci 2011: 7 (3): 165-173

31. Franzoni F, Quiñones-Galvan A, Regoli F, Ferrannini E, Galetta F. A comparative study of the in vitro antioxidant activity of statins. Int J Cardiol 2003; 90: 317-321.

32. Dursun S, Çuhadar S, Köseoğlu M, Atay A, Aktaş SG. The antiinflammatory and antioxidant effects of pravastatin and nebivolol in rat aorta. Anadolu Kardiyol Derg 2014; 14 (3): 229-233.

33. Büyükhatipoğlu H, Sezen Y, Yildiz A et al. Effects of statin use on total antioxidant capacity and ceruloplasmin activity. Clin Invest Med 2010; 33: 313-320.

34. Bauer AJ, Banek CT, Needham $K$ et al. Pravastatin attenuates hypertension, oxidative stress, and angiogenic imbalance in rat model of placental ischemia-induced hypertension. Hypertension. 2013; 61 (5): 1103-1110.

Received January 2, 2017. Accepted January 19, 2017. 